\title{
Evidence for a Strong Topological Insulator Phase in $\mathrm{ZrTe}_{5}$
}

\author{
G. Manzoni, ${ }^{1}$ L. Gragnaniello, ${ }^{2,}{ }^{*}$ G. Autès, ${ }^{3,4}$ T. Kuhn, ${ }^{2}$ A. Sterzi, ${ }^{1}$ F. Cilento, ${ }^{5}$ M. Zacchigna, ${ }^{6}$ V. Enenkel, ${ }^{2}$ \\ I. Vobornik, ${ }^{6}$ L. Barba, ${ }^{7}$ F. Bisti, ${ }^{8}$ Ph. Bugnon, ${ }^{3}$ A. Magrez, ${ }^{3}$ V. N. Strocov ${ }^{8}$ H. Berger, ${ }^{3}$ O. V. Yazyev, ${ }^{3,4}$ \\ M. Fonin, ${ }^{2}$ F. Parmigiani, ${ }^{1,5,9}$ and A. Crepaldi ${ }^{3,5, \dagger}$ \\ ${ }^{1}$ Universitá degli Studi di Trieste, Via Alfonso Valerio 2, Trieste 34127, Italy \\ ${ }^{2}$ Department of Physics, University of Konstanz, 78457 Konstanz, Germany \\ ${ }^{3}$ Institute of Physics, Ecole Polytechnique Fédérale de Lausanne (EPFL), CH-1015 Lausanne, Switzerland \\ ${ }^{4}$ National Centre for Computational Design and Discovery of Novel Materials MARVEL, \\ Ecole Polytechnique Fédérale de Lausanne (EPFL), CH-1015 Lausanne, Switzerland \\ ${ }^{5}$ Elettra-Sincrotrone Trieste S.C.p.A., Strada Statale 14, km 163.5, Trieste I-34149, Italy \\ ${ }^{6}$ Officina dei Materiali (IOM)-CNR, Laboratorio TASC, Area Science Park - Basovizza, I-34149 Trieste, Italy \\ ${ }^{7}$ Institute of Crystallography, CNR, Area Science Park, Strada Statale 14, km 163.5 Trieste I-34149, Italy \\ ${ }^{8}$ Swiss Light Source, Paul Scherrer Institut, CH-5232 Villigen, Switzerland \\ ${ }^{9}$ International Faculty, University of Köln, 50937 Köln, Germany
}

(Received 15 April 2016; revised manuscript received 22 October 2016; published 30 November 2016)

\begin{abstract}
The complex electronic properties of $\mathrm{ZrTe}_{5}$ have recently stimulated in-depth investigations that assigned this material to either a topological insulator or a 3D Dirac semimetal phase. Here we report a comprehensive experimental and theoretical study of both electronic and structural properties of $\mathrm{ZrTe}_{5}$, revealing that the bulk material is a strong topological insulator (STI). By means of angle-resolved photoelectron spectroscopy, we identify at the top of the valence band both a surface and a bulk state. The dispersion of these bands is well captured by $a b$ initio calculations for the STI case, for the specific interlayer distance measured in our x-ray diffraction study. Furthermore, these findings are supported by scanning tunneling spectroscopy revealing the metallic character of the sample surface, thus confirming the strong topological nature of $\mathrm{ZrTe}_{5}$.
\end{abstract}

DOI: 10.1103/PhysRevLett.117.237601

The discovery of topological insulators (TIs), characterized by metallic spin-polarized surface states connecting the bulk valence and conduction bands [1], has stimulated the search for novel topological phases of matter [2-6]. $\mathrm{ZrTe}_{5}$ has recently emerged as a challenging system with unique, albeit poorly understood, electronic properties [7-18]. Magnetotransport [11], magnetoinfrared [13], and optical spectroscopy [14] studies describe $\mathrm{ZrTe}_{5}$ in terms of a 3D Dirac semimetal. Theoretical calculations have predicted its bulk electronic properties to lie in the proximity of a topological phase transition between a strong and a weak TI (STI and WTI, respectively), where only the former displays topologically protected surface states at the experimentally accessible (010) surface [10]. The monolayer is also computed to be a 2D TI [10] and scanning tunneling microscopy or spectroscopy (STM and STS) experiments suggest the existence of topologically protected states at step edges $[19,20]$. However, the unambiguous identification of the topological phase of $\mathrm{ZrTe}_{5}$ is still lacking.

In this Letter we report on the STI character of the bulk $\mathrm{ZrTe}_{5}$ by combining $a b$ initio calculations and multiple experimental techniques, at temperatures both above and below that of the resistivity peak, $T^{*} \sim 160 \mathrm{~K}[7-9,15]$. Angle-resolved photoelectron spectroscopy (ARPES) experiments in the ultraviolet (UV) and soft x-ray (SX) energy ranges reveal the presence of two distinct states at the top of the valence band (VB). On the basis of photonenergy-dependent studies, we ascribe the origin of these two states to the bulk and crystal surface, respectively. We have performed $a b$ initio calculations of the topological phase diagram of $\mathrm{ZrTe}_{5}$, as a function of the interlayer distance $b / 2$. Our measured band dispersion is in agreement with the calculations and it is consistent with the STI case for $b / 2=7.23 \pm 0.02 \AA$. This value has been confirmed for our specimen by x-ray diffraction (XRD) measurements. Furthermore, the 3D Dirac semimetal phase is not protected by crystalline symmetries, and it manifests only for the specific $b / 2=7.35 \AA$ at the boundary between the STI and the WTI phases. Finally, STM-STS experiments confirms the metallic character of the surface termination of the bulk $\mathrm{ZrTe}_{5}$. Altogether our results indicate that $\mathrm{ZrTe}_{5}$ is a STI both above and below $T^{*}$, in proximity to the WTI phase.

High-quality $\mathrm{ZrTe}_{5}$ single crystals were grown by vapor transport technique [21]. The UV ARPES measurements were carried out at the APE beam line at the Elettra synchrotron, at $\sim 210 \mathrm{~K}$ with linear horizontal $(\mathrm{LH})$ polarization. The SX ARPES experiments were carried out at the ADDRESS beam line [22] at the Swiss Light Source, Paul Scherrer Institute, Switzerland, for both LH and linear vertical (LV) polarizations. In this set of measurements the 
sample temperature was kept at $\sim 15 \mathrm{~K}$ in order to suppress the Debye-Waller-like reduction of the coherent spectral weight [23]. Single crystal XRD measurements were performed in the temperature range $100-300 \mathrm{~K}$, for $8.85-\mathrm{keV}$ photon energy at the Elettra storage ring XRD1 beam line. The STM and STS experiments were performed on in situ-cleaved samples in a UHV chamber equipped with an Omicron Cryogenic STM, operating in constant-current mode at $10 \mathrm{~K}$. For more informations about the ARPES and STM and STS experimental conditions see the Supplemental Material [24-30]. Ab initio calculations were carried out, for the crystal lattice parameters taken from Ref. [31], within the density-functional theory (DFT) framework employing the generalized gradient approximation as implemented in the QUANTUM ESPRESSO package [32]. Spin-orbit effects were accounted for using fully relativistic norm-conserving pseudopotentials [33].

Figure 1(a) shows the $\mathrm{ZrTe}_{5}$ orthorombic crystal structure, with $\mathrm{Cmcm}$ (63) space group symmetry. The $\mathrm{ZrTe}_{5}$ natural cleavage plane results from two $\mathrm{ZrTe}_{3}$ chains oriented along the $a$ crystallographic direction. Each chain is formed by prisms with $\mathrm{Zr}$ (teal) and $\mathrm{Te}_{a}$ and $\mathrm{Te}_{d}$ (black) atoms at the apex and at the base of the prism, respectively. The chains are connected by $\mathrm{Te}_{z}$ atoms along the $c$ axis, with $a=3.99 \AA$ and $c=13.73 \AA$, as determined by x-ray powder diffraction [31]. Each crystal cell contains two

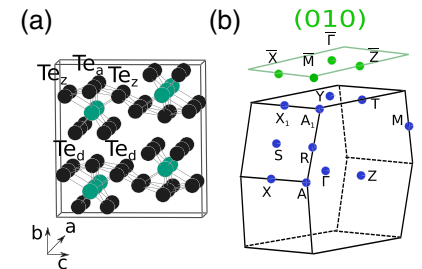

(c)

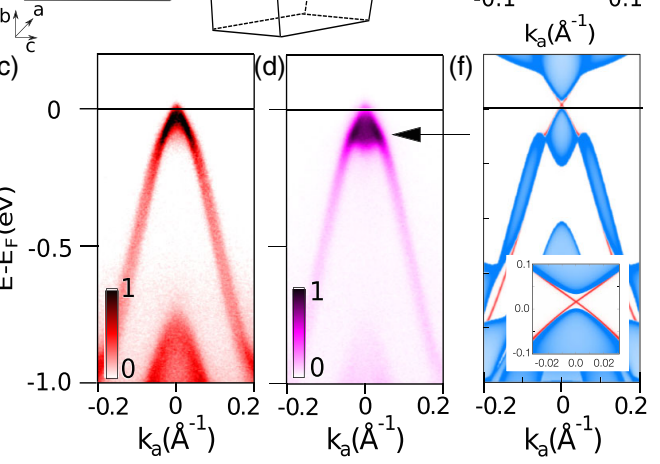

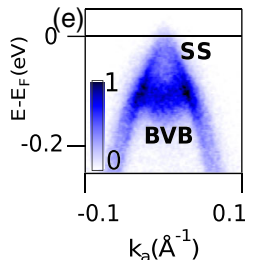

FIG. 1. (a) and (b) Crystal structure and 3D Brillouin zone, respectively, of $\mathrm{ZrTe}_{5}$. (c) and (d) UV ARPES measurements along the chain direction $k_{a}$ for 23.5 and $29 \mathrm{eV}$, respectively, corresponding to $\Gamma X$ and $Y X_{1}$. At $Y$ two states are observed, one crossing $E_{F}$ and the other forming an $M$-like shape reaching its maximum below $E_{F}$. (e) Zoom at $E_{F}$ measured at $22 \mathrm{eV}$ where the two states at the top of VB are more clearly resolved. (f) Calculated momentum resolved bulk density of states projected on the (010) surface (blue) and momentum resolved surface density of states at the (010) surface (red), for $b / 2=7.23 \AA$. The surface state is more clearly visible in the zoom at $E_{F}$ in the inset.
$\mathrm{ZrTe}_{5}$ planes piled along the $b$ axis. For this reason we indicate the interlayer distance with $b / 2$, whose value of $7.25 \AA$ reported in the literature [31] well matches our experimental finding, as it will be described later. Figure 1(b) shows the corresponding 3D Brillouin zone (BZ), along with the projection onto the investigated (010) surface.

Figures 1(c) and 1(d) show the UV ARPES measurements along the chain direction, $k_{a}$, for two photon energies corresponding to the $\Gamma X$ and $Y X_{1}$ high-symmetry directions of the 3D BZ, respectively. In Fig. 1(b), the VB disperses almost linearly at the $\Gamma$ point, with a small charge carriers density at the Fermi level $\left(E_{F}\right)$. In Fig. 1(d), we observe additional spectral weight for $E-E_{F}=0.1 \mathrm{eV}$, for wave vectors smaller than that of the linearly dispersing state, as indicated by a black arrow. The first state still disperses linearly across $E_{F}$. The second state reaches its maximum below $E_{F}$, and its dispersion deviates from linear, forming an $M$-like shape. The dispersion of two distinct states is better conveyed by Fig. 1(e). It shows a zoom at $E_{F}$ for a different wave vector orthogonal to the sample surface $k_{b}$, whose value is indicated by color lines in Figs. 3(a) and 3(b).

Two states are clearly resolved at the top of the VB. We denote one of these as the bulk valence band (BVB), as it displays a $k_{b}$ dependence evolving from a linear dispersion at the $\Gamma$ point to an $M$-like shape at the $Y$ point. The second state disperses linearly crossing $E_{F}$ with no appreciable photon energy dependence. Hence, its 2D character is consistent with the behavior of a surface state (SS). The presence of two nearly degenerate states at the top of the BVB has been reported both above and below $T^{*}$ (see the Supplemental Material [24]).
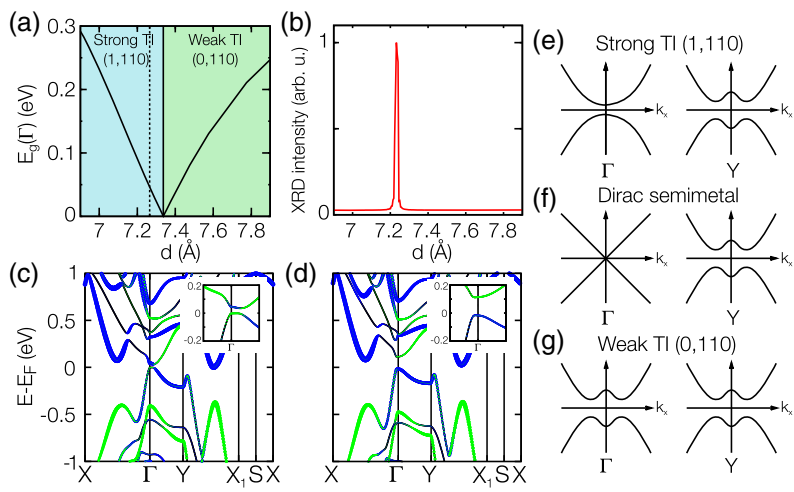

FIG. 2. (a) Band gap at $\Gamma$ as a function of the interlayer distance d. (b) X-ray diffraction intensity measured at $300 \mathrm{~K}$. (c) and (d) Calculated band structure of bulk $\mathrm{ZrTe}_{5}$ for the experimental interlayer distance $b / 2=7.23 \AA$ and an enlarged interlayer distance of $7.5 \AA$, respectively. Green and blue colors indicate the weight of the states on the $\mathrm{Te}_{d}$ and $\mathrm{Te}_{z} p$ orbitals. The inset shows a close-up in the bulk gap region at $\Gamma$. (e)-(g) Schematic band diagram at $\Gamma$ and $Y$ in the $k_{a}$ direction for (e) the STI phase, (f) the Dirac semimetal phase, and (g) the WTI phase. 
These results shed new light on two recent transport studies $[16,17]$. The former shows two states with semiconducting and semimetallic behaviors [16]; the latter proposes the simultaneous existence of surface massless and bulk massive Dirac particles in $\mathrm{ZrTe}_{5}$ [17].

The measured band structure is well reproduced by ab initio calculations, as shown in Fig. 1(f). The projected bulk band structure (blue area) captures the evolution of the BVB from linear to the $M$-like shape. More importantly, a surface state (red line) disperses within the band gap, as enhanced in the inset by zooming in on a small region across $E_{F}$.

When ad hoc measured crystal structure parameters are used, DFT calculations predict that $\mathrm{ZrTe}_{5}$ is a STI. When the spin-orbit coupling is not taken into account, conduction and valence bands are degenerate along a nodal line in the $k_{y}=0$ plane. Spin-orbit coupling breaks this degeneracy, resulting in a STI phase with a band inversion at $Y$. The product of parities of the valence-band states at the $Y$ point $k_{Y}=\left(\frac{1}{2}, \frac{1}{2}, 0\right)$ is reversed with respect to the other time-reversal invariant momentum (TRIM) $k$ points, which indicates that the $Z_{2}$ topological invariants are $(1,110)$. Upon increasing the interlayer distance $b / 2$, the gap at $\Gamma$ decreases, closes, and reopens [see Fig. 2(a)] in a phase where the product of parities of the valence states at $\Gamma$ is reversed and $\mathrm{ZrTe}_{5}$ is a WTI with invariants $(0,110)$. The observation of a topological phase transition is in agreement with the results in Ref. [10]. At the transition between the weak and strong phases, for $b / 2=7.35 \AA$, the gap at $\Gamma$ is closed and $\mathrm{ZrTe}_{5}$ lies in a Dirac semimetal phase. However, it is of paramount importance to note that this Dirac semimetal phase requires a fine-tuning of the interlayer distance. Figure 2(b) shows the results of our $\mathrm{x}$-ray diffraction study at $300 \mathrm{~K}$. The experimental value of the interlayer distance $b / 2=7.23 \pm 0.02 \AA$. The interlayer distance monotonically decreases as the temperature is lowered in the range from 300 to $100 \mathrm{~K}$ (see the Supplemental Material [24]). This is consistent with a previous XRD study [31], indicating that the $\mathrm{ZrTe}_{5}$ sample investigated in our work lies in the STI phase at temperatures both above and below $T^{*}$.

The band structure of $\mathrm{ZrTe}_{5}$ in the strong phase, obtained from the experimental structure, and in the weak phase, as obtained for a strained structure with $b / 2=7.5 \AA$, are shown in Figs. 2(c) and 2(d), respectively. The band inversion taking place at $\Gamma$ during the topological phase transition can be highlighted by computing the weight of the states on the $p$ orbitals of the $\mathrm{Te}_{d}$ and $\mathrm{Te}_{z}$ atoms, indicated by blue and green colors in Figs. 2(c) and 2(d), respectively. The three topological phases differ by their dispersion along the $k_{a}$ direction at the TRIM points $\Gamma$ and $Y$, as illustrated by the schematic band diagrams in Figs. 2(e)-2(g). While for each phase the dispersion around $Y$ is $M$ shaped, the dispersion around $\Gamma$ changes from parabolic in the strong topological phase to be $M$ shaped, reflecting the presence of a band inversion, in the weak topological phase.

In order to correctly determine the dimensionality of the SS and BVB, we carried out a photon-energy-dependent study of the $\mathrm{ZrTe}_{5}$ band structure. Figures 3(a) and 3(b) shows two constant energy maps (CEMs) in the plane formed by the chain direction, $k_{a}$, and the surface orthogonal, $k_{b}$. Similar results for the $\left(k_{a}, k_{c}\right)$ planes are discussed in the Supplemental Material [24]. The CEMs are extracted at -130 and $-50 \mathrm{meV}$, respectively. These values correspond to energies immediately below [Fig. 3(a)] and above [Fig. 3(b)] the maximum of the BVB at the $Y$ point, as shown by the black and blue lines traced on the ARPES image of Figs. 3(c) and 3(d). In Figs. 3(a) and 3(b), dashed colored lines indicate the positions of the band dispersion shown in Fig. 1 and Figs. 3(c) and 3(d). In Fig. 3(a) and (b), green lines guide the eyes of the readers on the calculated BVB for negative $k_{a}$. In Fig. 3(a) we identify two contours, but at this energy the BVB shows a weak dispersion along $k_{b}$. The dispersion of the SS is more clearly revealed in
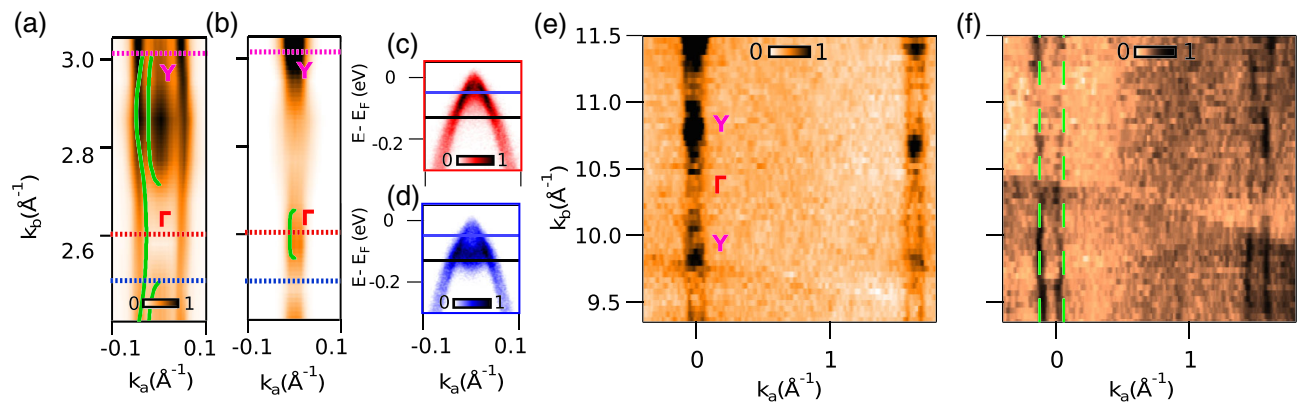

FIG. 3. Photon-energy-dependent study of $\mathrm{ZrTe}_{5}$. (a),(b) UV ARPES CEM in the $\left(k_{a}, k_{b}\right)$ plane. The CEMs correspond to -130 and $-50 \mathrm{meV}$, respectively. For negative $k_{a}$, green lines guide the eyes of the readers on the calculated BVB. (c) and (d) ARPES images with black and blue lines indicating the energy of the CEM of panels (a) and (b). In panel (b), a 2D state with open contour is clearly resolved from the calculated BVB. (e) and (f) CEM from the SX ARPES study with LV and LH polarization, respectively. (e) BVB contours with 3D bulk dispersion are visible, reproducing the results of (a). (f) Only open contours associated to the SS are visible, thus indicating the different symmetries of the BVB and SS. Green dashed lines indicate the 2D open contour of the surface state in (f). 
Fig. 3(b). In fact, at this binding energy, calculations show that the BVB is located around $\Gamma$, while we experimentally resolve an open contour with no dispersion along the entire $\mathrm{BZ}$, which we attribute to the SS.

The longer photoelectron mean free path in SX ARPES provides a more accurate mapping of $3 \mathrm{D}$ periodicity of the BVB dispersion [34], as shown in Fig. 3(e), displaying the SX photon energy scan for LV polarization. In this data set the binding energy of the CEM is $-50 \mathrm{meV}$, which corresponds to the same energy position of Fig. 3(a) with respect of the band structure, owing to the temperaturedependent energy shift of the band structure $[15,20]$ (see Supplemental Material [24]). We resolve clearly the BVB state with 3D dispersion, with three replicas along $k_{b}$ well reproducing the data of Fig. 3(a). BVB follows the expected periodicity and no signature of additional folding due to weak lattice distortion along the $b$ axis is observed [18].

Furthermore, it is interesting to notice the different origins of the BVB and the SS, which is ensured by their different response to the photon polarization. In fact, Fig. 3(f) shows a photon energy scan performed under the same experimental conditions of Fig. 3(e), but with LH polarization. The $3 \mathrm{D}$ bulk pockets associated to the BVB are not visible anymore and only the open contours associated to the SS are still present, indicated by the green dashed lines. According to the SX ARPES geometry, this effect can be interpreted as a consequence of the different symmetries of the BVB and SS, the former being even and the latter odd, with respect to the $\mathrm{ZrTe}_{5}$ symmetry plane. The different matrix element effects between the UV and SX ARPES results reflect the two different experimental geometries (see the Supplemental Material [24]).

We completed the occupied band structure mapping by performing STM and STS investigations. This allows us to directly access both the occupied and unoccupied DOS, below and above $E_{F}$. High-resolution STM images show
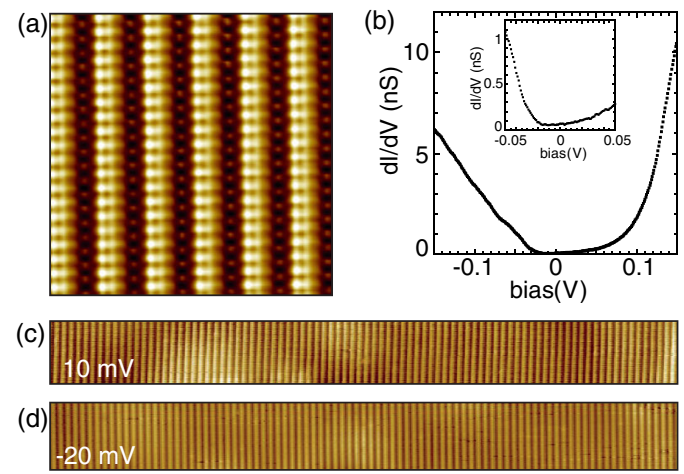

FIG. 4. (a) Atomic-resolution STM image of the $\mathrm{ZrTe}_{5}$ surface $(8 \mathrm{~nm} \times 8 \mathrm{~nm})$. Image conditions: $V=-300 \mathrm{mV}, I=300 \mathrm{pA}$. (b) $d I / d V$ spectrum on the $\mathrm{ZrTe}_{5}$ surface. Set point: $V=0.15 \mathrm{~V}$, $I=0.3 \mathrm{nA}, V_{\text {mod }}=5 \mathrm{mV}$. Inset: detail of the same spectrum, plotted in the $\pm 50 \mathrm{mV}$ range. (c) and (d) STM topography image of the $\mathrm{ZrTe}_{5}$ surface $(120 \mathrm{~nm} \times 13 \mathrm{~nm})$ recorded at $V=10 \mathrm{mV}$ and $V=-20 \mathrm{mV}$, respectively, $I=250 \mathrm{pA}$. the atomic structure of the $\mathrm{ZrTe}_{5}$ surface as chains of protruding pairs, separated by apparently monoatomic lines, as depicted in Fig. 4(a). According to our ARPES results, the top of the BVB lies in the proximity of $E_{F}$. Hence, in order to obtain insight about the conductivity in the bulk band gap, we performed STS in a low bias-voltage range. Figure 4(b) presents the $d I / d V$ spectrum in the $\pm 150 \mathrm{meV}$ range. The negative energy values correspond to the occupied states. Within this range, the curve appears to be characterized by a monotonic decrease and increase of conductivity with a minimum at around $-15 \mathrm{mV}$, as is clearly seen in the inset of the Fig. 4(b), which represents a plot of the same spectrum in the $\pm 50 \mathrm{mV}$ range.

Remarkably, within our experimental resolution, the conductivity of the sample never vanishes. To further elaborate on the latter point, we performed STM imaging at very low voltage bias, as depicted in Figs. 4(c) and 4(d). Both below $(-20 \mathrm{mV})$ and above $(+10 \mathrm{mV}) E_{F}$, we are able to image the atomic stripes of the $\mathrm{ZrTe}_{5}$ structure, even over areas which are larger than $100 \mathrm{~nm}$. Furthermore, the observation of standing waves in the $d I / d V$ conductance maps in proximity of step regions (see the Supplemental Material [24]) suggests that the metallic DOS at $E_{F}$ arises from a two-dimensional surface state. These observations rule out the interpretation of our ARPES data in terms of a replica of the bulk gapped band, as reported by a recent ARPES study [18].

In conclusion, in this Letter we report a comprehensive experimental and theoretical investigation of the electronic and structural properties of the bulk $\mathrm{ZrTe}_{5}$. We show that $\mathrm{ZrTe}_{5}$ is a bulk STI both above and below the temperature of the resistivity peak $T^{*}$. It lies near a topological phase transition to a WTI phase that can be triggered by an increase of the interlayer distance. This observation reconciles the recent conflicting results about the topological character of $\mathrm{ZrTe}_{5}$ [11-20]. Importantly, $\mathrm{ZrTe}_{5}$ is not a 3D Dirac semimetal, as this phase is not protected by crystalline symmetry and can only be realized precisely at the topological phase transition. Nonetheless, the band dispersion is close to a 3D Dirac semimetal, thus explaining the recent optical and electron transport measurements [11-14,16,17]. The close vicinity to the topological phase transition suggests the possibility of engineering the topological phase by a small variation of the interlayer distance, entering in the WTI phase. This can be achieved by changing the concentration of defects, which may vary depending on the growth conditions; this thus also accounts for the experimental observation of different $T^{*}$ in different studies $[11,15,35,36]$. The topological phase transition may also be achieved in a controlled way, either via alkali metal intercalation, as exploited for decoupling a single layer of $\mathrm{MoS}_{2}$ [37], or via chemical substitution. This will require further ARPES investigations of ad hoc grown samples with controlled doping and/or defect density. Finally, the observed monotonic decrease of $b / 2$ as a function of 
temperature suggests that, once the WTI phase is achieved, the transition to the STI phase can be controlled by temperature; this thus makes $\mathrm{ZrTe}_{5}$ a versatile platform for spintronics applications.

We gratefully acknowledge fruitful discussions with Marco Grioni and Doriano Lamba. This work was supported in part by the Italian Ministry of University and Research under Grants No. FIRBRBAP045JF2 and No. FIRB-RBAP06AWK3 and by the European Community Research Infrastructure Action under the FP6 "Structuring the European Research Area" Program through the Integrated Infrastructure Initiative "Integrating Activity on Synchrotron and Free Electron Laser Science," Contract No. RII3-CT-2004-506008. G. A. and O. V. Y. acknowledge support by the NCCR Marvel and the ERC starting grant "TopoMat" (Grant No. 306504). First-principles calculations have been performed at the Swiss National Supercomputing Centre (CSCS) under Project No. s675. F. B. acknowledges support by the SNF through Project No. 200021_146890. This work has been partly performed in the framework of the nanoscience foundry and fine analysis (NFFA-MIUR Italy) project. A. C. acknowledges financial support by the SNF.

*luca.gragnaniello@uni-konstanz.de †alberto.crepaldi@epfl.ch

[1] M. Z. Hasan and C. L. Kane, Rev. Mod. Phys. 82, 3045 (2010).

[2] S.-Y. Xu et al., Science 332, 560 (2011).

[3] M. Sakano, K. Okawa, M. Kanou, H. Sanjo, T. Okuda, T. Sasagawa, and K. Ishizaka, Nat. Commun. 6, 8595 (2015).

[4] Z. K. Liu et al., Nat. Mater. 13, 677 (2014).

[5] S.-Y. Xu et al., Science 347, 294 (2015).

[6] G. Autés et al., Nat. Mater. 15, 154 (2016).

[7] E. F. Skelton, T. J. Wieting, S. A. Wolf, W. W. Fuller, D. U. Gubser, T. L. Francavilla, and F. Levy, Solid State Commun. 42, 1 (1982).

[8] T. E. Jones, W. W. Fuller, T. J. Wieting, and F. Levy, Solid State Commun. 42, 793 (1982).

[9] T. M. Tritt, N. D. Lowhorn, R. T. Littleton IV, A. Pope, C. R. Feger, and J. W. Kolis, Phys. Rev. B 60, 7816 (1999).

[10] H. Weng, X. Dai, and Z. Fang, Phys. Rev. X 4, 011002 (2014).

[11] Q. Li, D. E. Kharzeev, C. Zhang, Y. Huang, I. Pletikosic, A. V. Fedorov, R. D. Zhong, J. A. Schneeloch, G. D. Gu, and T. Valla, Nat. Phys. 12, 550 (2016).

[12] Y. Zhou et al., Proc. Natl. Acad. Sci. U.S.A. 113, 2904 (2016).

[13] R. Y. Chen, Z. G. Chen, X.-Y. Song, J. A. Schneeloch, G. D. Gu, F. Wang, and N. L. Wang, Phys. Rev. Lett. 115, 176404 (2015).
[14] R. Y. Chen, S. J. Zhang, J. A. Schneeloch, C. Zhang, Q. Li, G. D. Gu, and N.L. Wang, Phys. Rev. B 92, 075107 (2015).

[15] G. Manzoni et al., Phys. Rev. Lett. 115, 207402 (2015).

[16] J. Niu, J. Wang, Z. He, C. Zhang, X. Li, T. Cai, X. Ma, S. Dja, D. Yu, and X. Wu, arXiv:1511.09315.

[17] A. Pariari and P. Mandal, arXiv:1603.05175v1.

[18] L. Moreschini, J. C. Johannsen, H. Berger, J. Denlinger, C. Jozwiak, E. Rotenberg, K. S. Kim, A. Bostwick, and M. Grioni, Phys. Rev. B 94, 081101 (2016).

[19] X.-B. Li et al., Phys. Rev. Lett. 116, 176803 (2016).

[20] R. Wu et al., Phys. Rev. X 6, 021017 (2016).

[21] F. Lévy and H. Berger, J. Cryst. Growth 61, 61 (1983).

[22] V. N. Strocov, X. Wang, M. Shi, M. Kobayashi, J. Krempasky, C. Hess, T. Schmitt, and L. Patthey, J. Synchrotron Radiat. 21, 32 (2014).

[23] J. Braun, J. Minar, S. Mankovsky, V. Strocov, N. Brookes, L. Plucinski, C. M. Schneider, C. S. Fadley, and H. Ebert, Phys. Rev. B 88, 205409 (2013).

[24] See Supplemental Material at http://link.aps.org/ supplemental/10.1103/PhysRevLett.117.237601 for more details about the photon- and polarization-dependent photoemission results, the theoretical calculations, and the STM results.

[25] D. N. McIlroy, S. Moore, D. Zhang, J. Wharton, B. Kempton, R. Littleton, M. Wilson, T. M. Tritt, and C. G. Olson, J. Phys. Condens. Matter 16, L359 (2004).

[26] A. Damascelli, Phys. Scr. T109, 61 (2004).

[27] A. A. Mostofi, J. R. Yates, G. Pizzi, Y. S. Lee, I. Souza, D. Vanderbilt, and N. Marzari, Comput. Phys. Commun. 185, 2309 (2014).

[28] A. Umerski, Phys. Rev. B 55, 5266 (1997).

[29] Y. Kim, B. J. Wieder, C. L. Kane, and A. M. Rappe, Phys. Rev. Lett. 115, 036806 (2015).

[30] C. Fang, Y. Chen, H.-Y. Kee, and L. Fu, Phys. Rev. B 92, 081201(R) (2015).

[31] H. Fjellvaggg and A. Kjejshus, Solid State Commun. 60, 91 (1986).

[32] P. Giannozzi, S. Baroni, N. Bonini, M. Calandra, R. Car, C. Cavazzoni, D. Ceresoli, G. L. Chiarotti, M. Cococcioni, I. Dabo et al., J. Phys. Condens. Matter 21, 395502 (2009).

[33] A. Dal Corso and A. Mosca Conte, Phys. Rev. B 71, 115106 (2005).

[34] V. N. Strocov, J. Electron Spectrosc. Relat. Phenom. 130, 65 (2003).

[35] W. Yu, Y. Jiang, J. Yang, Z. L. Dun, H. D. Zhou, Z. Jiang, P. Lu, and W. Pan, arXiv:1602.06824.

[36] S. Okada, T. Sabongi, and M. Ido, J. Phys. Soc. Jpn. 49, 839 (1980).

[37] T. Eknapakul, P. D. C. King, M. Asakawa, P. Buaphet, R.-H. He, S.-K. Mo, H. Takagi, K. M. Shen, F. Baumberger, T. Sasagawa et al., Nano Lett. 14, 1312 (2014). 